\section{(2) OPEN ACCESS}

\title{
Novel pleural-bladder pump in malignant pleural effusions: from animal model to man
}

\author{
Philippe Astoul, ${ }^{1}$ Sophie Laroumagne, ${ }_{1}^{1}$ Jeroen Capel, ${ }^{2}$ Nicholas A Maskell ${ }^{3,4}$
}

${ }^{1}$ Department of Thoracic Oncology, Pleural Diseases and Interventional Pulmonology, Assistance Publique-Hôpitaux de Marseille (AP-HM), Hopital Nord, Marseille, France

${ }^{2}$ Sequana Medical AG, Zurich, Switzerland

${ }^{3}$ North Bristol Lung Centre, Southmead Hospital, Bristol, UK ${ }^{4}$ Academic Respiratory Unit, Department of Clinical Sciences, Bristol University, Bristol, UK

\section{Correspondence to}

Professor Philippe Astoul,

Department of Thoracic

Oncology, Pleural Diseases and Interventional Pulmonology, Assistance Publique-Hôpitaux de Marseille (AP-HM), Hopital Nord, Marseille 13915, France; pastoul@ap-hm.fr

Received 22 August 2019 Revised 8 February 2020 Accepted 14 February 2020

Published Online First

12 March 2020

Check for updates

(C) Author(s) (or their employer(s)) 2020. Re-use permitted under CC BY-NC. No commercial re-use. See rights and permissions. Published by BMJ.

To cite: Astoul P,

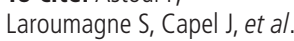

Thorax 2020:75:432-434.

\section{ABSTRACT}

Malignant pleural effusion is common and causes disabling symptoms such as breathlessness. Treatments are palliative and centred around improving symptoms and quality of life but an optimal management strategy is yet to be universally agreed. A novel pump system, allowing fluid to be moved from the pleural space to the urinary bladder, may have a role for the management of recurrent malignant pleural effusion. We hereby describe the first animal study using this device and the results of the first application in patients.

\section{BACKGROUND}

Malignant pleural effusion (MPE) is common and causes disabling symptoms such as breathlessness. Treatments are palliative and centred around improving symptoms and quality of life but an optimal management strategy is yet to be universally agreed. The recent European Respiratory Society clinical statement on MPE recommend indwelling pleural catheter insertion for selected patients and talc pleurodesis when aiming for pleural symphysis. ${ }^{1}$

A novel pump system may have a role for the management of recurrent malignant pleural effusion. The Alfapump system is a subcutaneously implanted battery-powered device allowing ascitic fluid to be automatically moved from the peritoneal cavity to the urinary bladder before being eliminated through normal urination. The safety and efficacy of the Alfapump has already been evaluated in cirrhotic liver patients. ${ }^{2}$ The modified Pleurapump system is a subcutaneous device with a rechargeable battery, allowing fluid to be moved from the pleural space to the urinary bladder. The pleurapump system (figure 1a) consists of an implantable sealed housing (A) containing an internally powered pump with supportive electronic components and circuits; two implantable silicone catheters with polyester cuffs to anchor them in position-a pleural catheter $(15 \mathrm{Fr}, 80 \mathrm{~cm}$; B) and a bladder catheter (15Fr, $44 \mathrm{~cm} ; \mathrm{C})$ - and a non-implantable charger (D and $\mathrm{E})$. One of the catheters is located within the pleural space and the other is implanted in the urinary bladder, with the pump and battery implanted subcutaneously into the abdomen. The device moves the fluid from the pleural space to the bladder via the pump. The pumping algorithms control fluid pumping in accordance with the predetermined parameters and is monitored by pressure and position sensors. The implanted battery may be recharged transcutaneously using a wireless charger which can also programme the pump and collect performance data.
We describe the first animal study using this device and the results of the first human study using this device.

\section{The animal study}

The Pleurapump was successfully implanted into four pigs according to the European Convention for the Protection of vertebrate animals ${ }^{3}$ (figure 1b). The first procedure took over 1 hour with subsequent procedures being complete in less than $40 \mathrm{~min}$. Successful fluid transport cycles were completed in three animals. In one animal, successful fluid transport cycles were not possible due to technical problems priming the pump prior to implantation and subsequent trapping of air within the catheters. Reasons for unsuccessful pump cycles included insufficient fluid present in the pleural cavity, insufficient transport time and excessive bladder pressure. Pressure-time curves during pump activation at different respiratory rates in the absence and presence of fluid in the pleural cavity indicate that successful and comparable pump functioning and fluid transport was achieved at the different respiratory conditions tested (figure 2). There were no complications with any of these insertions.

\section{First in human: phase 1 study}

Two patients were enrolled in this study from Marseille, France (NCT01952327) ${ }^{4}$ (figure 1c). Both underwent a medical thoracoscopy for the pleural access. ${ }^{5}$ The pump was placed in a subcutaneous pocket on the right side of the abdomen. The subcutaneous pocket was created by blunt dissection via a small incision of approximately $5 \mathrm{~cm}$ in length. Through a small incision $(2$ and $3 \mathrm{~cm}$ ) the pleural and the bladder catheter were inserted. Insertion of the catheter into the pleural space was done during a thoracoscopy procedure. An introducer kit was employed to facilitate the placement (Carefusion, Vernon Hills, Illinois, USA). The bladder catheter was placed at the same time by an experienced doctor or surgeon familiar with the placement of suprapubic catheters. Insertion of the catheter into the bladder was performed percutaneously using the Seldinger technique using an introducer kit. Both catheters were tunnelled subcutaneously to the pump pocket so no component protrudes above the skin. An anaesthetist was present throughout the procedure to control pain and in case of any requirement for sedation. Both the pleural and bladder catheters were cut to a custom length prior to attachment to the pump. The Dacron discs attached to the pump encourage the growth of tissue around the pump, to anchor it in place within the implantation pocket. The clinician 

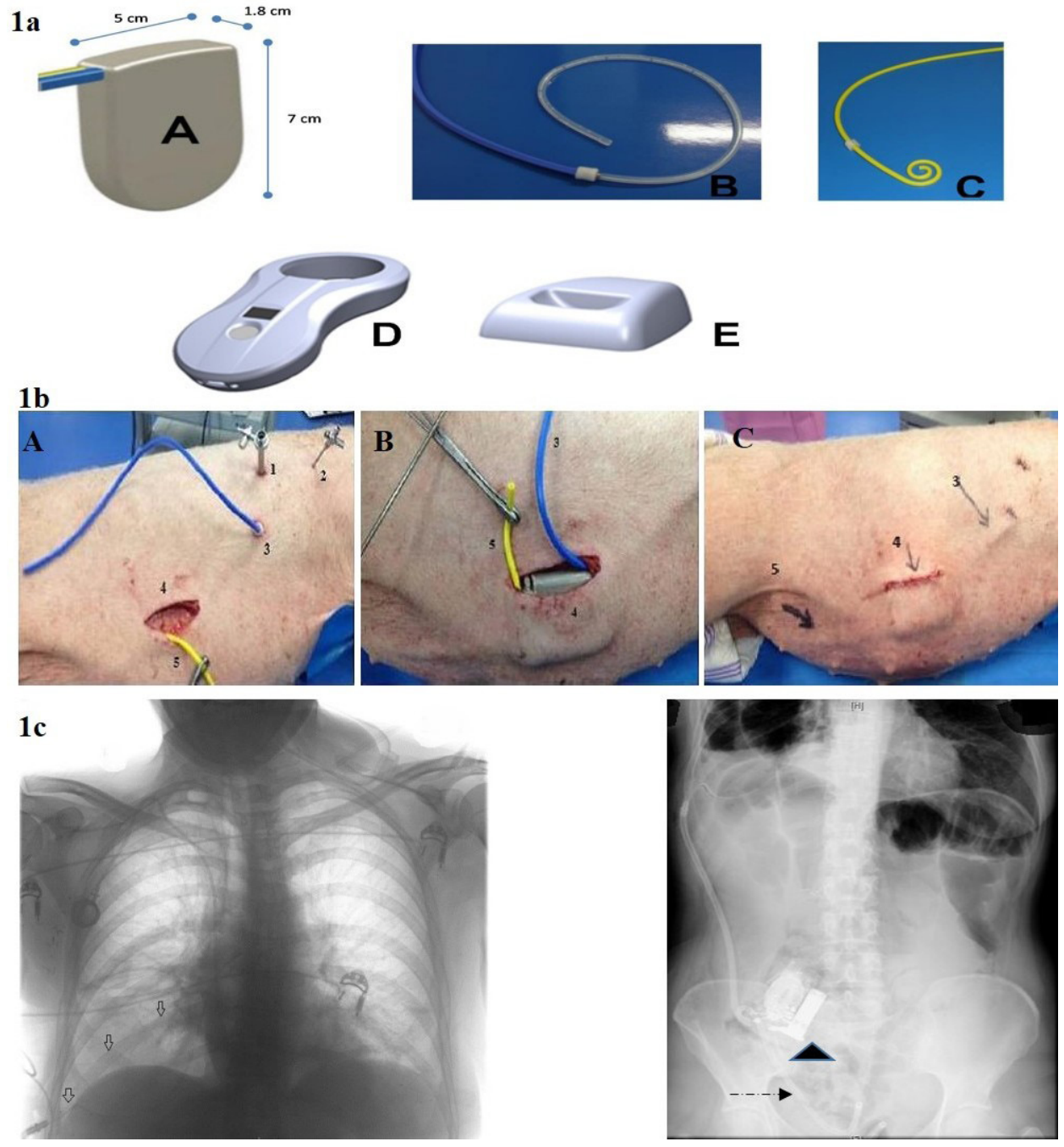

Figure 1 The pleurapump system. (1a) The pleurapump system components—-pleurapump (A), pleural catheter (B), bladder catheter (C), smart charger (D) and docking station (E). (b) Surgical implantation of the pleurapump (1.7 mm pleural trocar; $2.2 \mathrm{~mm}$ pleural needle; 3. pleural catheter; 4. pump pocket; 5 . bladder catheter). (A) Pleural access $(1,2,3)$ and subcutaneous pocket $(4)$ with yellow bladder catheter (B) nləurapump placement (pleural catheter in blue) (C) Pleurapump in place after the end of the procedure. (c) Patient's imaging with implanted device. $\checkmark$ Pleural catheter; -.-B Bladder catheter; Implanted pleurapump).

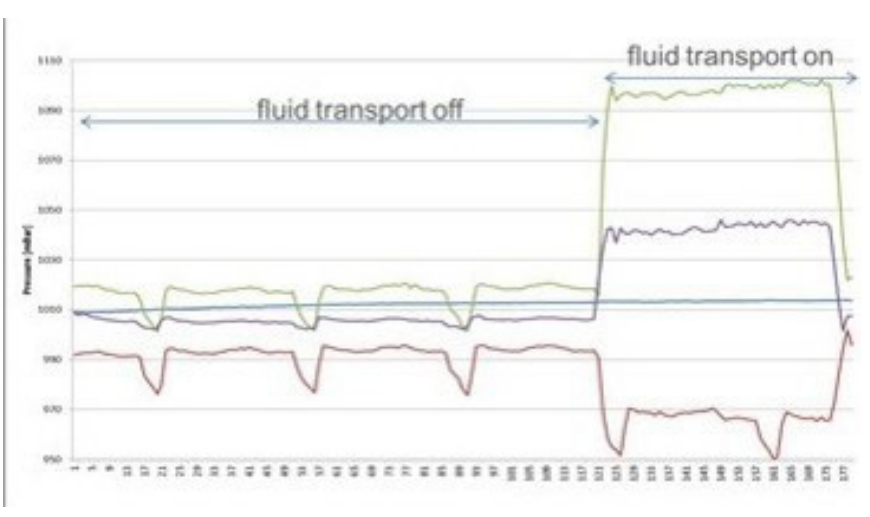

Figure 2 Pressure-time curve during pump activation in the absence and presence of fluid in the pleural cavity. Blue line: ambient pressure (mBar), red line: inlet pressure (mBar), green line: pump pressure (mBar), purple line: outlet pressure (mBar). verifies the function of the pump after implantation and prior to incision closure. Thereafter, the clinician can wirelessly retrieve operational information from the implanted pump and make adjustments to treatment using the Charger.

The first patient was a 50-year-old woman suffering from a primary lung adenocarcinoma. She was enrolled in the trial after developing recurrent malignant pleural effusion leading to six therapeutic thoracentesis, each aspiration was greater than $1000 \mathrm{~mL}$. A previous attempt for talc pleurodesis had failed. The pleural fluid was very bloody with a lot of clot. The patient had the device implanted during medical thoracoscopy lasting $70 \mathrm{~min}$ followed. The pump and pleural catheter were placed on the right side and $760 \mathrm{~mL}$ of fluid was drained during the procedure. Prophylactic antibiotics were administered prior to the procedure, and the pump was started 12 hours after the implantation at a rate of $100-300 \mathrm{~mL} /$ day. The patient was discharged 4 days after implant. Following discharge the patient underwent chemotherapy, and due to underlying disease progression and side effects of chemotherapy was admitted 1 month after the implant. Her chemotherapy was 
stopped and she received parenteral nutritional support resulting in stabilisation of her general condition.

The patient then began suffering symptoms of breathlessness on day 40 and was admitted on day 55. Investigation revealed fibrin blockage of the pleural catheter and subsequent malfunction of the pump. The catheter was replaced on day 61 and full recovery achieved after 7 days (day 68). The patient died 3 months after device placement due to disease progression. During the patient's therapy the pleurapump removed a total of $17.1 \mathrm{~L}$ of pleural fluid at rates as high as $600 \mathrm{~mL} /$ day. Visual Analogue Scale (VAS) assessment for abdominal pain remained unchanged at 0 throughout the treatment, chest or thoracic pain reduced from 3.9 to 0 by day two and remained at 0 for the remaining time. Breathlessness pain assessed by combined VAS decreased gradually from nine at baseline to 5 , however, as complications related to underlying disease and catheter blockage occurred, this again rose to 9.7 at month 2 .

The second patient was a 45 -year-old woman who was diagnosed thirteen years before with breast cancer initially treated by surgery and, due to a relapsed disease 4 years later with chemotherapy, radiotherapy and hormone therapy. Further progression after 5 years of follow-up led to a radical mastectomy, adjuvant chemotherapy and hormone therapy. A new malignant pleural effusion due to progression of the disease led to enrolment in the clinical trial.

The patient was implanted during medical thoracoscopy lasting $85 \mathrm{~min}$. The pump and pleural catheter were placed on the right side and $300 \mathrm{~mL}$ of fluid was drained during the procedure. Prophylactic antibiotics were administered. The pump was initially set to pump $100-300 \mathrm{~mL} /$ day and the patient was discharged 4 days after implant. The patient's pleural effusion was managed for 12 months using the pleurapump with a total of $33.5 \mathrm{~L}$ removed by pump without the need for additional pleural intervention. The patient presented with dyspnoea on day 35 following implant, related to moderate recurrence of right pleural effusion attributed to suspected pleural catheter occlusion and demonstrable chemotherapy-related asthenia. However the patient improved spontaneously within 72 hours without any additional intervention. VAS assessment for abdominal pain remained effectively unchanged at 0 throughout the 12 months treatment. Chest or thoracic pain decreased from 3.2 to 0 immediately following implant and remained low (0-1.1) with transient increases to 3.9 at 2 months and again rising to 6.1 at 9 months before again falling to 0 at 12 months. Breathlessness pain by combined VAS decreased gradually from 7.5 at baseline to levels between 1 and 2.2, with temporal increases to 3.7 at 3 months and 7.0 at 12 months.

\section{DISCUSSION}

We have shown that the pleurapump can be successfully implanted into pigs and humans using standard medical and surgical techniques. The procedure can be conducted by chest physicians with experience in the placement of pleural drains with assistance from urologists familiar with the placement of supra-pubic catheters using a modified Seldinger technique followed by subcutaneous tunnelisation of the catheter to the subcutaneous abdominal pocket for the pump. It is vital for the catheters' functionality to be aligned in the same plane toward the pump.

This system looks to address the issues represented by administration of an intrapleural agent to obtain a pleural symphysis or by the insertion of a long-term chest drain or indwelling pleural catheter. For the latter, some patients find the frequent need for medical or nursing intervention to be intrusive, and drainages can be painful, especially in cases with trapped lung. In addition, in several countries, the cost-efficacy of such devices, mainly the pre-filled vacuum bottles, is not relevant.

Occasional peritoneal catheter blockage has been previously observed in earlier studies using the same pump system (alfapump). ${ }^{2}$ In cirrhotic ascites this problem had largely been solved with the change to the newer Medionics catheter. ${ }^{7}$ Both of our patients had this catheter placed and still suffered a blockage, 1 requiring reintervention and the other resolved spontaneously. Blockages and catheter malfunctions are also noted as a complication in indwelling pleural catheters (IPC), ${ }^{8-10}$ often requiring intervention. Regarding the pleurapump safety, the small samples size makes it difficult to assess other risks as pleural or bladder/urinary infections, potential retrograde flow, cancer cell dissemination which will be a major issue in future clinical trials.

In conclusion, the pleurapump was able to effectively remove fluid from the pleural cavity under both controlled physiological and spontaneous breathing conditions and to stop pumping in the absence of fluid. Further clinical studies enrolling large cohort of patients are needed to validate the place of pleurapump in the management of patients presenting recurrent malignant pleural effusions.

Acknowledgements We are grateful to Charles-Hugo Marquette (Université Côte d'Azur, Nice, France) and Gary YC Lee (University of Western Australia, WA, Australia) for their advice and support during advisory board meetings with the company. We also acknowledge Pr S. Berdah (LBA-UMRT24, C.E.R.C., Aix-Marseille University, Marseille, France) and his team for their skilled technical assistance during the experimental study.

Funding This study was sponsored by Sequana Medical AG.

Competing interests PA and NAM were part of the scientific advisory board for Sequana Medical AG for this study. JC is a member of Sequana Medical. NAM has received unrestricted research grants from $B D$ and Rocket Medical to run indwelling pleural catheters RCT's.

Patient consent for publication Not required.

Provenance and peer review Not commissioned; externally peer reviewed.

Open access This is an open access article distributed in accordance with the Creative Commons Attribution Non Commercial (CC BY-NC 4.0) license, which permits others to distribute, remix, adapt, build upon this work non-commercially, and license their derivative works on different terms, provided the original work is properly cited, appropriate credit is given, any changes made indicated, and the use is non-commercial. See: http://creativecommons.org/licenses/by-nc/4.0/.

\section{REFERENCES}

1 Bibby AC, Dorn P, Psallidas I, et al. ERS/EACTS statement on the management of malignant pleural effusions. Eur Respir J 2018;52:1800349.

2 Bellot P, Welker M-W, Soriano G, et al. Automated low flow pump system for the treatment of refractory ascites: a multi-center safety and efficacy study. $J$ Hepatol 2013;58:922-7.

3 Council of Europe. European convention for the protection of vertebrate animals used for experimental and other scientific purposes. Strasbourg, 18III 1986.

4 Investigation into the automated drainage of recurrent effusions from the pleural space in thoracic malignancy (ADEPT), 2014. Available: http://clinicaltrialsgov/show/ NCT01952327

5 Skalski JH, Astoul PJ, Maldonado F. Medical thoracoscopy. Semin Respir Crit Care Med 2014:35:732-43.

6 Bureau C, Adebayo D, Chalret de Rieu M, et al. Alfapump® system vs. large volume paracentesis for refractory ascites: a multicenter randomized controlled study. J Hepatol 2017;67:940-9.

7 Solbach P, Höner Zu Siederdissen C, Wellhöner F, et al. Automated low-flow ascites pump in a real-world setting: complications and outcomes. Eur J Gastroenterol Hepatol 2018:30:1082-9.

8 Van Meter MEM, McKee KY, Kohlwes RJ. Efficacy and safety of tunneled pleural catheters in adults with malignant pleural effusions: a systematic review. J Gen Intern Med 2011;26:70-6.

9 Davies HE, Mishra EK, Kahan BC, et al. Effect of an indwelling pleural catheter vs chest tube and talc pleurodesis for relieving dyspnea in patients with malignant pleural effusion: the TIME2 randomized controlled trial. JAMA 2012;307:2383-9.

10 Sabur NF, Chee A, Stather DR, et al. The impact of tunneled pleural catheters on the quality of life of patients with malignant pleural effusions. Respiration 2013;85:36-42. 